\title{
Impact of Varying Radio Power Density on Wireless Communications of RF Energy Harvesting Systems
}

\author{
Yu Luo*, Lina $\mathrm{Pu}^{\dagger}$, Lei Lei ${ }^{\ddagger}$ \\ * Department of Electrical and Computer Engineering, Mississippi State University, Mississippi State, MS, 39759 \\ ${ }^{\dagger}$ Department of Computer Science, University of Alabama, Tuscaloosa, AL 35487 \\ ${ }^{\ddagger}$ SnT, University of Luxembourg, 29 Av. J.F. Kennedy, Luxembourg \\ Email:yu.luo@ece.msstate.edu, lina.pu@ua.edu, lei.lei@uni.lu
}

\begin{abstract}
Through field experiments, we observed a varying instantaneous charging capacity of the energy buffer with respect to the dynamic intensity of the incident RF signal in the RF energy harvesting system (RF-EHS). The dependency of charging capacity on the incident power of RF signal challenges existing RF energy harvesting models that assume constant charging capacity. In order to accurately describe the energy harvesting process in the real system, we propose a new energy clamp model. The new model reveals that RF intensity higher than the sensitivity of the harvester circuit cannot always guarantee successful energy reception, especially when the energy level of energy buffer is high while the intensity of the incident power is relatively weak. In order to improve the efficiencies of energy harvest and energy utilization in an RF-EHS, we develop new offline (i.e., non-causal) optimal and online (i.e., causal) suboptimal data transmission strategies based on the energy clamp model. Simulation results show that the new strategy can considerably improve the throughput after taking into account the varying instantaneous charging capacity caused by the dynamic RF power density in the air.
\end{abstract}

Index Terms-RF energy harvesting system, varying RF power density, energy clamp model, transmission strategy

\section{INTRODUCTION}

Applications of the smart city, precision agriculture, public safety, and surveillance usually require the participation of numerous sensors and lower-power actuators, which have strong demands on energy efficiency and self-sustainability. Radio frequency (RF) energy harvesting is one of the promising technologies to achieve those goals. Through scavenging ambient RF power radiated from TV towers, WiFi access points, and cellular base stations, an RF energy harvesting system (EHS) is able to drive low-power wireless devices in the long term without battery replacement [1].

However, an EHS needs to use the collected energy very efficiently to optimize the communication performance due to the thin RF power density in the environment [2], [3]. Along with the development of data transmission strategies that aim to improve the energy utilization subject to different constraints such as the energy causality [4], the size of a data buffer [5], and the limited capacity of an energy buffer [6], [7], hardware issues of the RF energy harvesting system are being more and more considered in recent works to make the transmission strategy realistic in real applications [8]-[10].

In existing RF energy harvesting models, it is commonly accepted that if the aging effect on electronic materials is ignored, the instantaneous amount of energy that an energy buffer can be charged to (i.e., instantaneous charging capacity) is a constant [11], [12]. According to this assumption, RF waves arriving at an EHS can always be successfully harvested as long as the strength of incoming energy is stronger than the sensitivity ${ }^{1}$ of an energy harvesting circuit.

In this article, we challenge the above common assumption and show through experimental results that in the RF energy harvester, the instantaneous charging capacity of energy buffer fluctuates in the real RF-EHS. The output voltage of the voltage multiplier, which we call the charging voltage, is not constant, but varies with the intensity of the RF signal as shown in Fig. 1. In supercapacitors, the instantaneous charging capacity is a quadratic function of the charging voltage, (i.e., $\left.E_{\max }^{i}=\frac{1}{2} C\left(V_{\max }^{i}\right)^{2}\right)$, and thereby will change with the power density of RF energy in a practical RF energy harvester. The varying instantaneous charging capacity will lead to the following situation: the RF energy may not be successfully harvested even if the RF power density is stronger than the sensitivity of the harvester circuit. It happens when the buffered energy, $E_{r}^{i}$, in the supercapacitor is higher than the instantaneous charging capacity, $E_{\max }^{i}$. This phenomenon indicates that existing RF energy harvesting models may not match real systems well, which will inevitably result in a low performance of existing data transmission strategies or even cause them infeasible in reality.

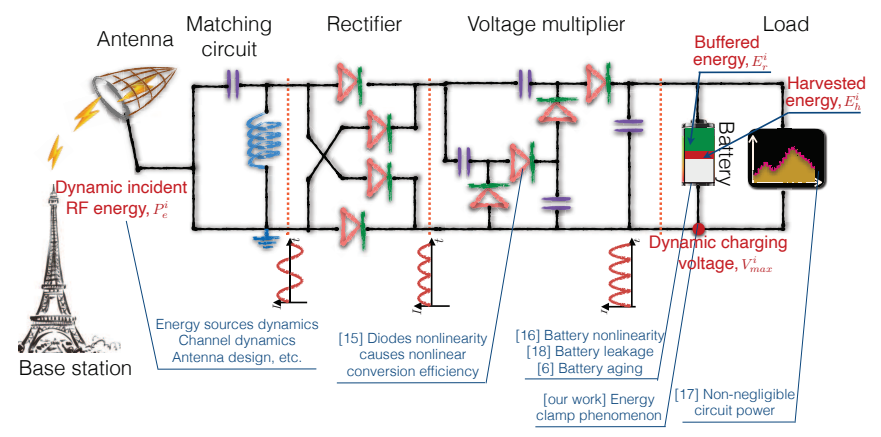

Figure 1: A diagram of RF energy harvesting system.

After taking into account varying instantaneous charging capacity, we propose an energy clamp model to accurately

\footnotetext{
${ }^{1}$ The power sensitivity is the minimum strength of incident power that an energy harvester can receive. The power sensitivity of an ambient RF energy harvesting circuit is usually between $-20 \mathrm{dBm}$ and $-9 \mathrm{dBm}$ [13].
} 
describe the scenario when RF energy cannot be harvested (i.e., energy clamped) due to the reduced charging capacity. In the proposed model, the maximal charging capacity of RFEHS is not only determined by the hardware design (e.g., the circuit configuration and the type of energy buffer) but also affected by the RF strength. In addition, several important hardware properties, such as the nonlinear charging of energy buffer and the unidirectional conductivity of diodes in the circuit, are also considered to make the new model practical.

An essential difference between the new energy clamp model and the conventional model [6], [14] is how to estimate the amount of harvested energy, $E_{h}^{i}$, in transmission scheduling. In the conventional model, the energy packet can be fully harvested as long as it is stronger than the sensitivity of the harvester circuit. Therefore, $E_{h}^{i}$ is usually calculated directly based on the size of the energy packet without considering the state of charge (SOC) of the energy buffer. In the energy clamp model, $E_{h}^{i}$ depends not only on the size of the energy packet but also on the varying charging capacity and the SOC of the energy buffer. The interaction between the charging and discharging processes is fully considered. Compared with existing work, the energy clamp model can accurately calculate the amount of energy that can be harvested from the environment, thereby making the scheduled data transmission realistic.

Another obvious difference comes from the shape of the feasible energy tunnel in the transmission scheduling. In conventional models, the width of the feasible energy tunnel (i.e., the instantaneous charging capacity) is constant. A tunnel of fixed width is only valid for a system like solarbased harvester, which generates a stable charging voltage regardless of changes in the environment. In the new model, the instantaneous charging capacity changes with the intensity of the incident RF wave. Therefore, the width of the energy tunnel is different in different cycles.

Due to the energy clamp phenomenon, the design of an offline (i.e., non-causal) optimal transmission strategy becomes a challenging issue since the energy harvesting process and the data transmission strategy can affect each other. The design of offline optimal transmission strategy needs to know the future $E_{h}^{i}$ to better plan the energy usage for throughput maximization; the transmission strategy itself, however, can affect the energy harvesting process through managing the SOC or buffered energy of the energy storage. Accordingly, we need to jointly optimize the transmission strategy and harvest scheme rather than simply optimizing the former based on the predetermined value of latter, which is another difference between our work and existing data transmission strategies.

To summarize, the contributions of our work are threefold. 1) Through experimental research on the RF-EHS, we identify the energy clamp phenomenon, and further verify this phenomenon through theoretical analysis. It it verified that the intensity of incident RF power can significantly affect the instantaneous charging capacity of battery in an RF energy harvester. To the best of our knowledge, this is the first work identified the varying charging capacity and the energy clamp phenomenon in RF-EHS.

2) We propose a new model to correctly describe the energy harvesting process considering the energy clamp phenomenon in a real RF-EHS. Due to the energy clamp feature, traditional offline optimal transmission strategies that are based on conventional EH model no longer work. We developed new offline optimal transmission policy that have energy clamp phenomenon considered.

3) Compared with the conventional model overlooking the dynamic instantaneous charging capacity, the energy clamp model achieves about $16 \%$ higher throughput in offline optimal transmission scheduling when the relative standard deviation of the charging voltage is $17.9 \%$. Inspired by the behavior of offline optimal solution, we use a prediction based online (i.e., causal) suboptimal strategy as an example to verify that online policies based on the energy clamp model are feasible in reality.

The rest of this paper is organized as follows. Section II introduces the related work. The impact of varying power density on the instantaneous charging capacity of energy buffer is analyzed in Section III. We describe the new energy harvesting model in Section IV and propose an offline optimal transmission strategy in Section V. The performance of the offline optimal and online suboptimal transmission strategies are evaluated in Section VI and Section VII, respectively. Conclusions are drawn in Section VIII.

\section{RELATED WORK}

Considering the limited energy supply, it is critically important to efficiently utilize the harvested energy for better communication throughput in RF-EHS networks. For this purpose, many offline optimal power management strategies are designed to investigate the optimal communication throughput that can be achieved with the limited energy supply. Although the offline approach that assumes to know the future energy arrivals is non-causal thus not feasible in real systems, it provides a benchmark solution as well as insights into efficient transmission scheduling, which will be discussed in Section VII. Next, we introduce energy harvesting models and transmission strategies that are most relevant to our work.

In a classic energy harvesting model, it is assumed that RF energy arrives at an EHS as discrete energy packets with varying sizes (i.e., predetermined amount of energy contained) and random time of arrivals. Therefore, the accumulative energy received by a harvester is represented as a staircase curve. It is proven in [6] that the design of an offline optimal transmission strategy can be converted into a piecewise-linear optimization problem. Considering the size constraint of energy buffer, the profile of accumulative energy consumption for data transmissions must stay within a feasible energy tunnel bounded by the accumulative harvested energy and buffer size. It is also pointed out that from a graphical perspective, the profile of the optimal energy consumption should be the tightest string in the tunnel in order to maximize the throughput. 
To make the transmission strategy realistic, more and more hardware features and practical issues in real RF-EHSs are considered in recent studies. In [15], the influences of the diodes nonlinearity and the circuit parasitic on the energy harvesting process are investigated. The results show that, due to diodes nonlinearity and the circuit parasitic, the efficiency of energy conversion in the RF harvesting circuit varies depending on the input power density of the RF signal. In [16], it is verified that the amount of energy harvested from a given RF energy packet is not a constant but depends on the SOC of an energy buffer. This phenomenon results from the nonlinear charging feature of the energy buffer. In our work, we justify that the amount of harvested energy is not only affected by the nonlinear battery, but also determined by the energy clamp phenomenon as discussed in Section IV-B.

The circuit power is another hardware feature that needs to be considered in the power management. Besides the energy spent on the RF transceiver, an EHS inevitably consumes extra energy to power the microprocessor, digital-to-analog converter (DAC), and other operating modules. After considering the overhead of the circuit, continuous transmissions of data becomes inefficient, while an on-off transmission strategy is advocated for higher throughput [17]. Battery leakage and aging issues are also investigated in the literature. Specifically, the energy stored in an energy buffer gradually leaks with time due to the off current in a circuit and the self-discharge characteristic of the energy buffer. If the energy leakage rate is fixed, it is equivalent to adding a constant operation power to the circuit [18]. Moreover, if the energy leakage is caused by the material aging, the width of the feasible energy tunnel will gradually reduce over time. The degradation of the battery's capacity is commonly slow but irreversible.

Although the practical issues such as hardware nonlinearity, battery nonlinearity, circuit power, and battery imperfection have been recognized and considered in the energy harvesting system, the explorations on the RF-EHS circuit and the RF environment are still insufficient. It is commonly assumed that the instantaneous charging capacity is constant or slowly degrades in the long run if battery aging is considered. However, in this work, we will point out that the instantaneous charging capacity has significant dynamics and varies with the power density of the RF signal. The assumption on constant charging capacity will cause inaccurate modeling of RF-EHS and lead to inefficient power management. In this work, we will justify the feature of dynamic charging capacity utilizing the experiment results collected from field tests. And we will propose an energy clamp model to accurately describe the energy harvesting process aiming to provide an accurate and realistic model for efficient data transmission design.

\section{The VARYing InStantaneous Charging CAPACity}

In this section, we first introduce the phenomenon of RF-EHS charging voltage variation caused by the dynamic strength of incident radio energy through experimental results. Afterwards, the variation of RF power density in environments is investigated. At last, we analyze the impact of varying RF power density on the instantaneous charging capacity.

\section{A. Dependency of Charging Voltage on RF Power Density}

The dependency of charging voltage on RF power density results from the hardware characteristic of RF-EHS. A common structure of RF-EHS consists of antenna, matching circuit, rectifier, voltage multiplier, and energy buffer $^{2}$ as depicted in Fig. 1. After being captured by the antenna, the RF signal will be rectified and boosted to a higher voltage to charge the energy buffer. The output of the voltage multiplier is not constant, but varies with the intensity of the incident power of the RF signal. When the energy buffer is fully filled, its capacitive reactance will be infinite; meanwhile, the energy buffer reaches the voltage of the voltage multiplier. For simplicity, the output voltage of voltage multiplier is referred to as the charging voltage, $V_{\max }$, which is the charging voltage of supercapacitor given the strength of the RF signal.

In order to verify how the strength of RF waves affects the charging voltage of RF-EHS, we conducted lab experiments, as demonstrated in Fig. 2. An HP 8648C signal generator [19] connected to a power amplifier serves as the energy source radiating $915 \mathrm{MHz} \mathrm{RF}$ signal at a controllable power density. A Powercast P2110-EVB energy harvesting board [20] stores the harvested energy to a $50 \mathrm{mF}$ supercapacitor (i.e., energy buffer). To estimate the strength of RF waves arrived at the harvester, a Keysight N9340B spectrum analyzer [21] is placed at a position symmetrical to the energy harvester. The spectrum analyzer and harvester are equipped with the same receiving antenna. In addition, multimeters are used to monitor the charging current and the voltage of the energy buffer ${ }^{3}$. By adjusting the output power of the signal generator, we can radiate RF signals with varying power density.

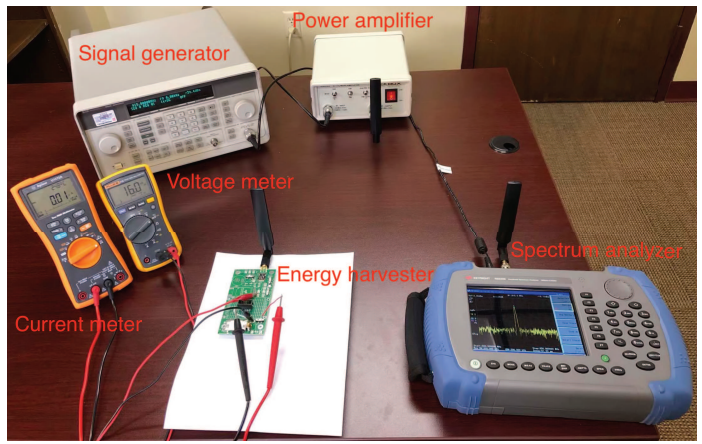

Figure 2: Experiment setup for RF energy harvesting.

In Fig. 3, we show the charging voltage of P2110 harvester with respect to the intensity of incident RF power. Some existing test results with other RF harvesters [22]-[24] are

\footnotetext{
${ }^{2}$ Supercapacitors have low leakage current and are the ideal power buffer between the energy harvester and the load. Therefore, we use supercapacitor as an example and refer energy buffer to supercapacitor throughout this paper.

${ }^{3} \mathrm{We}$ consider the energy buffer to be fully charged when the average charging current reduced to $1 \mu \mathrm{A}$ for a low charging voltage $(<1 \mathrm{~V})$ or $10 \mu \mathrm{A}$ for a relatively high charging voltage $(\geq 1 \mathrm{~V})$. The reason behind this setting is that it takes an infinite time to fully charge the energy buffer.
} 
also presented in the figure for validation and comparison purposes. From the figure, it can be observed that the charging voltage of RF harvesters varies with the strength of the RF signal. Taking P2110 harvester as an example, the charging voltage drops from $1.95 \mathrm{~V}$ to $1.57 \mathrm{~V}$ when the intensity of RF energy has only $1.5 \mathrm{dBm}$ difference (i.e., reducing from -7.0 $\mathrm{dBm}$ to $-8.5 \mathrm{dBm}$. The varying charging voltage is a common phenomenon of different harvesters as shown in Fig. 3.

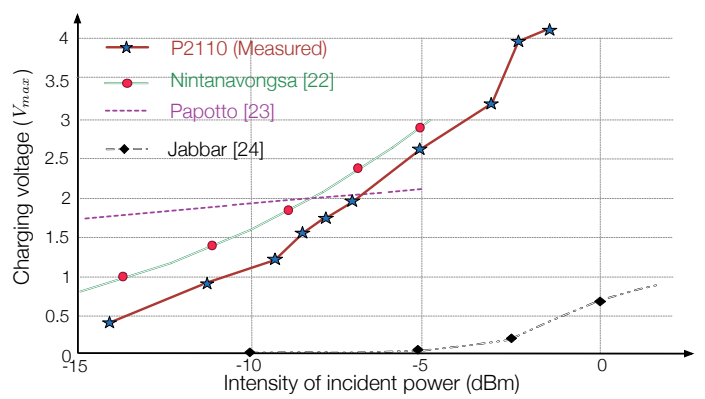

Figure 3: Charging voltages, $V_{\max }$, with respect to the incident power for different RF harvesters.

\section{B. Dynamic RF Power Density and Varying Charging Capac- ity in RF-EHS}

With the previous results, we have verified the dependency of RF-EHS's charging voltage on the RF power density caused by the hardware of RF-EHS. Next, we conducted outdoor experiments to examine the strength of ambient radio signals and the charging characteristic of RF-EHS in a real scenario.

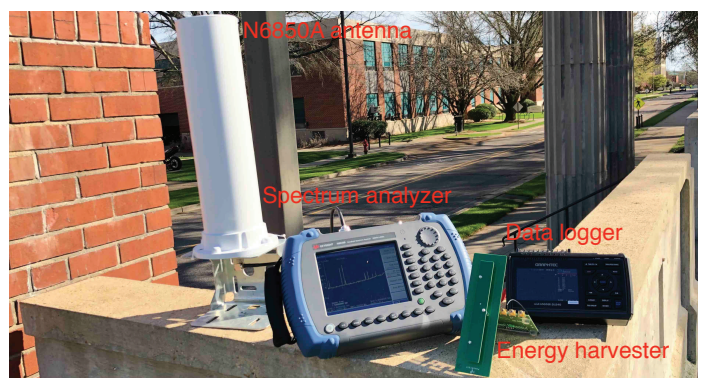

Figure 4: Experiment setup for RF environment monitoring and charging voltage measurement.

The field experiments were conducted on the campus of Mississippi State University. As shown in Fig. 4, a Keysight N6850A broadband omnidirectional antenna [25] was connected with the spectrum analyzer to monitor the RF environment from $30 \mathrm{MHz}$ to $3 \mathrm{GHz}$. The average gain of N6850A is between $0 \mathrm{dBi}$ and $-2 \mathrm{dBi}$ in the target frequency band. The sampling interval of spectrum analyzer was $1.15 \mathrm{~s}$. A Powercast P21XXCSR-EVB board was deployed to harvest RF energy from an LTE antenna array (not shown in Fig. 4) that is 280 feet away from the energy harvester. Fig. 5 demonstrates the spectrograms of ambient RF signal measured around the Powercast energy harvester. The $\mathrm{X}$-axis represents the frequency and the Y-axis is time, with the strength of RF signal in each $1 \mathrm{MHz}$ frequency band represented by color.
Table I: Mean value and standard deviations of signal strength.

\begin{tabular}{|c|c|c|c|c|}
\hline Frequency $(\mathrm{MHz})$ & 887.5 & 739 & $1,952.5$ & 2,115 \\
\hline$\mu(\mathrm{dB})$ & -10.4 & -16.8 & -30.2 & -33.8 \\
\hline$\sigma(\mathrm{dB})$ & $1.2^{*}$ & 2.6 & 2.8 & 2.8 \\
\hline
\end{tabular}

* The $1.2 \mathrm{~dB}$ standard deviation in signal strength seems stable from communication point of view, which, however, is strong enough to cause $17.9 \%$ charging voltage dynamics in the RF energy harvesting system.

The two strongest bands on $887.5 \mathrm{MHz}$ and $739 \mathrm{MHz}$ are the downlink signal from LTE base stations that are about 280 feet away. The eNB IDs of the two LTE base stations are 204758 and 204558, respectively [26]. The $1952.5 \mathrm{MHz}$ and $2115 \mathrm{MHz}$ bands are from other distant LTE base stations.

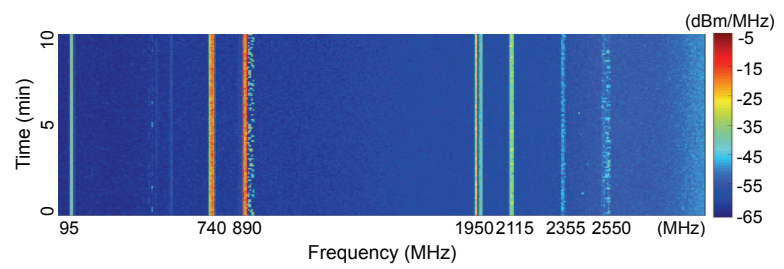

Figure 5: Spectrograms of ambient RF energy.

In Table I, we list the mean value $(\mu)$ and the standard deviation $(\sigma)$ of the radio power at different frequencies. From the table, it can be realized that the RF strength in an outdoor environment varies over time and the standard deviation of radio strength on $887.5 \mathrm{MHz}$ is $1.2 \mathrm{~dB}$. The significant fluctuations of the RF energy intensity in outdoor environments are also verified in Fig. 6 . The envelope of radio waves is presented to visualize the upper and lower limits of the energy strength. As can be observed from the figure, the instantaneous $\mathrm{RF}$ intensity has random and high fluctuations (from -13 to $-7 \mathrm{dBm}$ ) on $887.5 \mathrm{MHz}$ band. Note that, as reported in [27], the strength of RF waves in an indoor environment has even higher fluctuations than outdoor signal due to the shadowing effect caused by the large number of obstacles and potential scatterers. The dynamic intensity of ambient RF signal may result from a combined effect of small-scale fading, shadowing, and weather dependent attenuations [3].

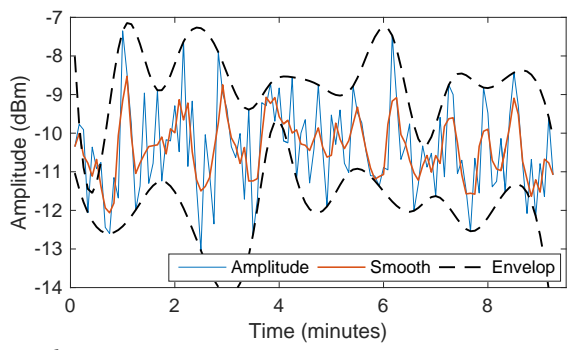

Figure 6: Intensity of ambient RF energy on $887.5 \mathrm{MHz}$.

To verify the impact of dynamic RF power density on the charging voltage of RF energy harvester, we used a Graphtec GL240 data logger to record the instant charging voltage (i.e., the output voltage of the voltage multiplier) every $10 \mathrm{~ms}$. In tests, we used two different antennas: a directional patch antenna with $6 \mathrm{dBi}$ gain at $915 \mathrm{MHz}$, and an omnidirectional 
dipole with $1 \mathrm{dBi}$ gain at the same frequency. The instant charging voltage of energy harvester is presented in Fig. 7.

As demonstrated in the figure, there are many impulses in the untreated curves (blue and yellow). This indicates that in a short time period, there are remarkable variations (i.e., more than $0.5 \mathrm{~V}$ ) on the harvester's charging voltage. According to the measurements, with $1 \mathrm{dBi}$ omnidirectional antenna, the standard deviation of charging voltages can achieve $0.12 \mathrm{~V}$, which is $17.9 \%$ of the average charging voltage. The dynamic range of charging voltage is from $0.4 \mathrm{~V}$ to $1.45 \mathrm{~V}$ with $1 \mathrm{dBi}$ omnidirectional antenna and from $1.0 \mathrm{~V}$ to $1.7 \mathrm{~V}$ with $6 \mathrm{dBi}$ directional antenna. Since the charging capacity of the supercapacitor is a quadratic function of charging voltage, the dynamic RF power density in the outdoor experiment resulted in 13.14 times and 2.89 times variation in the instantaneous charging capacity with $1 \mathrm{dBi}$ and $6 \mathrm{dBi}$ antenna, respectively.

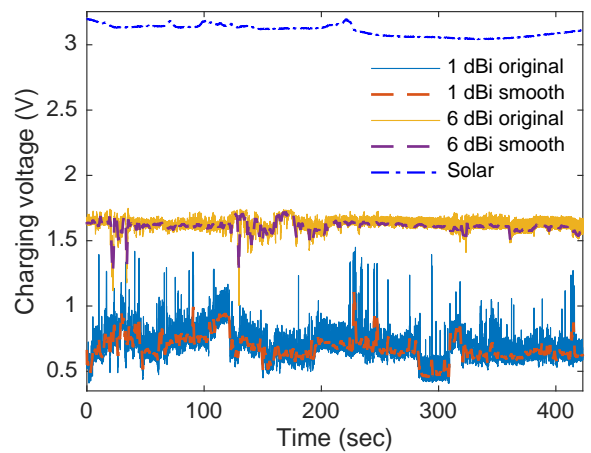

Figure 7: Charging voltage of RF and solar energy harvesters in real outdoor environments.

To show the long-term feature of charging voltages, we filter out impulses using a 100 points smooth filter and then plot the smoothed curves in Fig. 7. It can be observed that the dynamics of smoothed charging voltage are still significant. In addition, from the experimental results, we can observe that using directional antenna can significantly improve both the long-term and short-term stabilities of the charging voltage. Specifically, compared with the $1 \mathrm{dBi}$ omnidirectional antenna, the $6 \mathrm{dBi}$ directional antenna can reduce the standard deviation of charging voltages from $0.12 \mathrm{~V}$ to $0.06 \mathrm{~V}$ in a short term or $0.09 \mathrm{~V}$ to $0.04 \mathrm{~V}$ in a long-term.

It should be noted that a solar-powered EHS has nearly constant charging voltage. According to the electrical properties of amorton solar cells [28], the charging voltage of a single glass type cell is $0.89 \mathrm{~V}$ when the illuminance of natural light ranges from $10 \mathrm{k}$ to $100 \mathrm{k}$ lux in outdoor use. The charging voltage presented in Fig. 7 is charged with a solar energy harvester of four cells. During our outdoor experiment, the daylight intensity changed between 5.8 klux and 89.9 klux, the standard deviation of the charging voltage with Panasonic AM-1801CA [28], however, was only $1.3 \%$ of the mean value, as shown in Fig. 7. In addition to RF energy harvesters, the characteristic of the dynamic charging voltage also commonly exists in vibration- and thermal-based EHS [29], [30]. This prompted us to study the impact of varying charging voltage

on transmission scheduling. Although conclusions of this work may not be directly applicable to vibration- or thermal-based EHS, they can provide useful design principles for optimal transmission strategies in vibration- and thermal-based EHS.

In supercapacitors, the instantaneous charging capacity is a quadratic function of the charging voltage. The dynamic charging voltage will result in varying instantaneous charging capacity in RF-EHSs. The conventional RF energy harvesting models and transmission strategies assuming a constant instantaneous charging capacity is more suitable for solar-based EHS rather than RF-EHS, since this assumption is valid only when the charging voltage of the energy harvester is unaffected by the environment like in solar-powered EHSs. For an RF-EHS, the varying instantaneous charging capacity will inevitably affect the energy harvesting process. Therefore, we propose a new model to accurately calculate the amount of energy that can be truly harvested in a real RF-EHS, which will be presented in the next section.

\section{New EnERgy HARVESTING MODEL}

In this section, we propose an energy clamp model to accurately describe the variation of charging capacity in the energy harvesting process. Here, we focus on co-located RF-EHSs in which the energy harvester and the wireless transceiver share the antenna in a time-division manner; hence, the energy reception and the data communication cannot be performed at the same time. The impact of energy clamp feature on the offline optimal transmission scheduling is also discussed.

\section{A. Two Scenarios in Energy Harvesting Process}

To build a new energy harvesting model, we start by analyzing two energy harvesting scenarios as illustrated in Fig. 8. Both the exponential charging (i.e., red curve) and discharging (i.e., brown curve) processes of energy buffer (i.e., capacitors [31] are presented. We use $V^{t_{i}}$ to denote the instant voltage of the energy buffer at time $t_{i}$. According to the energy-voltage function of supercapacitor, the buffered energy at time $t_{i}$ is $E_{r}^{i}=\frac{1}{2} C\left(V^{t_{i}}\right)^{2} . P_{e}^{i}$ is the incident power density of the RF signal in the charging period $t_{i} \rightarrow T_{i}$. $V_{\max }^{i}$ (i.e., blue lines in Fig. 8) is the instantaneous charging voltage, i.e., the open-circuit voltage of the voltage multiplier, during $t_{i} \rightarrow T_{i}$. As verified in Fig. 3 of Section III, $V_{\max }^{i}$ is a variable that depends on $P_{e}^{i}$. We assume $P_{e}^{i}$ is constant within a harvesting interval $t_{i} \rightarrow T_{i}$. Next, we detail the two scenarios in Fig. 8.

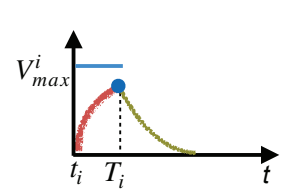

(a)

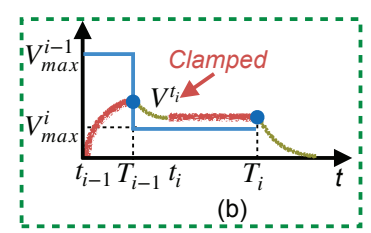

Figure 8: Schematic diagrams of energy harvesting process: (a) no energy clamp, (b) energy clamped.

a) $V^{t_{i}}<V_{\max }^{i}$ : The buffered energy, $E_{r}^{i}$, is lower than the instantaneous charging capacity, $E_{\max }^{i}$. In this situation, 
the voltage of the supercapacitor can be eventually charged to $V_{\max }^{i}$ with the unlimited charging time. We call it reached the instantaneous charging capacity. When the charging time is limited as $U_{i}=T_{i}-t_{i}$, the amount of energy to be harvested, $E_{h}^{i}$, can be calculated according to energy harvest equation, which is modeled in the next subsection. This situation is corresponding to Fig. 8 (a).

b) $V^{t_{i}} \geq V_{\text {max }}^{i}$ : When the buffered energy, $E_{r}^{i}$, in the supercapacitor is higher than or equal to the instantaneous charging capacity, $E_{\text {max }}^{i}$, the energy clamp happens. As with a low energy intensity, the incident RF energy can only charge the supercapacitor to $V_{\max }^{i}$, which is lower than the concurrent voltage of the supercapacitor. In this situation, the supercapacitor is "full" and no energy can be harvested even if the incident power of RF signal is stronger than the sensitivity of the harvester, the harvester cannot receive any RF energy if $V^{t_{i}} \geq V_{\max }^{i}$. However, whether the supercapacitor is "full" or not depends on the intensity of the incident RF energy. If at time $t_{i+1}$, $V^{t_{i}}=V^{t_{i+1}}<V_{\text {max }}^{i+1}$, the supercapacitor will not be "full" but can be potentially charged to a higher voltage. The energy clamp situation is corresponding to Fig. 8 (b).

The energy clamp phenomenon usually happens when RF power in the air was strong during pervious charging interval, $t_{i-1} \rightarrow T_{i-1}$ and becomes weak in the next charging interval, $t_{i} \rightarrow T_{i}$ (i.e., $P_{i-1}^{e} \gg P_{e}^{i}$ ), as illustrated in Fig. $8(\mathrm{~b})$. The blue line indicates the drop of $V_{\max }$. At time $t_{i}$, the voltage of supercapacitor is higher than the charging voltage, i.e., the red charging curve was above the blue line during $t_{i} \rightarrow T_{i}$. The voltage of the supercapacitor will be clamped at $V^{t_{i}}$ due to the unidirectional conductivity of diodes in the circuit.

The energy clamp situation illustrated in Scenariob) cannot be correctly represented in existing energy harvesting models. However, the energy clamp phenomenon eventually occurs in a frequent manner when the incident power density of RF signal drops due to dynamic RF environments. It can be realized that the energy arriving at a harvester cannot be successfully received if the current energy level is high while the strength of incoming RF energy is relatively weak.

The energy clamping phenomenon will dramatically change the behavior of RF energy harvester. For convenience, most of the existing works consider the energy arriving at a harvester as discrete packets containing random energy; the RF energy ${ }^{4}$ can always be harvested as long as the incident power of RF signal is higher than the sensitivity of the harvester circuit [4][6], [8]. This indicates that when Scenariob) happens, the existing models will falsely add an amount of energy that actually cannot be captured. Planing the data transmissions based on the energy that does not exist would make it infeasible for implementation.

The energy clamping feature of RF-EHS motivates us to propose a new energy harvesting model to accurately calculate

\footnotetext{
${ }^{4}$ In the conventional EH model, the amount of harvested energy is predetermined based on energy conversion efficiency and the size of energy packet.
}

the amount of energy that can be truly harvested by an EHS in a dynamic RF environment.

\section{B. Energy Clamp Model}

Since the harvester and transceiver share the same antenna in a time-division manner in the co-located EHSs, data transmission and energy harvest will not happen at the same time. As demonstrated in Fig. 9 (a), there are interleaved charging (i.e., red curve) and discharging (i.e., brown curve) processes.

In the discharging phase, when we schedule the optimal transmission strategy, only the amount of harvested energy in the charging phases matters and the charging interval is neglected. Therefore, after we seamed the discharging phases, the accumulation of harvested energy forms a staircase curve as demonstrated in the purple lines in Fig. 9(b) and (c). The rise height represents the amount of energy harvested in the charging process, $E_{h}^{i}$, and the tread depth equals to the length of the discharging phase.

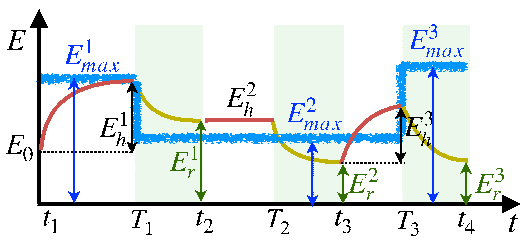

(a) Charging and discharging process

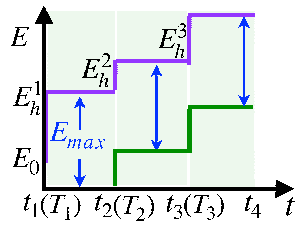

(b) Conventional $\mathrm{EH}$ model

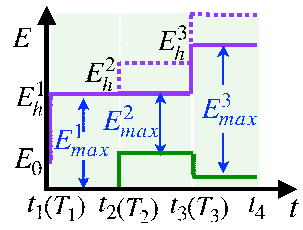

(c) Energy clamp model
Figure 9: (a) Schematic diagram of charging and discharging processes, where blue lines represent the instantaneous charging capacity, $E_{\text {max }}^{i}$, varying with dynamic RF power density, red curves show the energy charging process with $E_{h}^{i}$ as the amount of harvested energy and brown curves indicate energy discharging during data transmission. (b) Feasible energy tunnel in conventional EH model, where purple lines represent the accumulative harvested energy; green lines are obtained by shifting purple lines down by $E_{\max }$. (c) Energy tunnel in energy clamp model.

In the conventional $\mathrm{EH}$ model, we can obtain a feasible energy tunnel by shifting the purple staircase curve down by $E_{\max }$, as shown in Fig. 9 (b). The design of optimal transmission strategy is converted into planning the profile of accumulative energy consumption within the feasible energy tunnel for highest communication throughput. The fixed width of the tunnel indicates that the instantaneous charging voltage is assumed to be constant in the conventional model [4][6], [8]. In addition, it also ignores the energy clamping scenario in the real RF energy harvesting process and results in overestimated $E_{h}^{2}$ (i.e., EHS cannot harvest any energy in interval $t_{2} \rightarrow T_{2}$ ).

In the energy clamp model, by contrast, the variation of the instantaneous charging capacity, $E_{\text {max }}^{i}$, indicates its dependency on the incident power density of RF signal. When the 
buffered energy at the beginning of charging interval $t_{2} \rightarrow T_{2}$ is higher than $E_{\text {max }}^{2}$, the energy stored in the buffer is clamped and the harvester cannot receive energy from the environment in this charging interval (i.e., $E_{h}^{2}=0$ ), as indicated in Fig. 8 (b). When the energy buffer is not clamped, the EHS can charge the energy buffer to a higher voltage as shown in intervals $t_{1} \rightarrow T_{1}$ and $t_{3} \rightarrow T_{3}$. According to the charging curve of capacitor and the energy-voltage function [31], we have

$$
V_{t_{i}}=V_{\max }^{i}\left(1-e^{-\frac{\sum_{j=1}^{i} U_{j}}{R C}}\right), \quad E_{r}^{i}=\frac{1}{2} C\left(V_{t_{i}}\right)^{2} .
$$

Here, $U_{i}$ is the length of charging interval $t_{i} \rightarrow T_{i} \cdot \sum_{j=1}^{i} U_{j}$ represents the accumulative charging time. $R$ and $C$ are the resistance of the RC charging circuit and the capacitance of the energy buffer (i.e., supercapacitor), respectively.

When the energy clamp does not occur, the energy buffer will be charged to a higher voltage. $\Delta v_{i+1}$ and $E_{h}^{i+1}$ indicate the voltage and energy increments with charging time $U_{i+1}$, respectively. The new voltage and energy level become

$$
\begin{aligned}
& V_{t_{i+1}}=V_{t_{i}}+\Delta v_{i+1}=V_{\max }^{i+1}\left(1-e^{-\frac{\sum_{j=1}^{i+1} U_{j}}{R C}}\right), \\
& E_{r}^{i+1}=E_{r}^{i}+E_{h}^{i+1}=\frac{1}{2} C\left(V_{t_{i+1}}\right)^{2} .
\end{aligned}
$$

By solving (1) and (2), we get the charging function with no energy clamp as

$E_{h}^{i}=\mathcal{C}\left(E_{r}^{i-1}, U_{i}, E_{\text {max }}^{i}\right)=A_{i}^{1}\left(A_{i}^{2}\right)^{2}+A_{i}^{1} A_{i}^{3}\left(E_{r}^{i-1}\right)^{\frac{1}{2}}+A_{i}^{1} A_{i}^{4} E_{r}^{i-1}$

where

$$
\begin{array}{ll}
A_{i}^{1}=\frac{1}{2} e^{-\frac{2 U_{i}}{R C}}, & A_{i}^{2}=\left(2 E_{\max }^{i}\right)^{\frac{1}{2}}\left(e^{\frac{U_{i}}{R C}}-1\right), \\
A_{i}^{3}=2^{\frac{3}{2}} A_{i}^{2}, & A_{i}^{4}=2\left(1-e^{\frac{2 U_{i}}{R C}}\right) .
\end{array}
$$

Considering the energy clamp phenomenon, in case of $E_{r}^{i} \geq$ $E_{\max }^{i+1}$ (i.e., $V_{t_{i}} \geq V_{\max }^{i+1}$ ), the harvester cannot receive energy in the current harvesting cycle, i.e., $E_{h}^{i}=0$; otherwise, the received energy is calculated via $\mathcal{C}(\cdot)$. Therefore, we can use a step function to formulate $E_{h}^{i}$.

$$
E_{h}^{i}= \begin{cases}\mathcal{C}\left(E_{r}^{i-1}, U_{i}, E_{\text {max }}^{i}\right), & E_{r}^{i-1}<E_{\text {max }}^{i} \\ 0, & E_{r}^{i-1} \geq E_{\text {max }}^{i} .\end{cases}
$$

One essential difference between the new energy clamp model and the conventional $\mathrm{EH}$ model is the way that $E_{h}^{i}$ is estimated. In most of the conventional $\mathrm{EH}$ model, $E_{h}^{i}$ is only determined by the length of charging interval, $U_{i}$, which is modeled as a predetermined random sequence [4], [6], [14], [16]-[18]. In the energy clamp model, $E_{h}^{i}$ is also affected by the buffered energy, $E_{r}^{i-1}$, owing to the battery nonlinearity, and the variation of the charging capacity, $E_{\max }^{i}$ due to the energy clamp phenomenon. Compared with existing works, the energy clamp model is able to accurately estimate the amount of energy that can be harvested from the environment making the scheduled data transmission realistic. Another obvious difference comes from the shape of the energy tunnel. The tunnel of fixed width in conventional models is only valid for a system like solar-based harvester which generates a stable charging voltage regardless of changes in the environment. In the new model, the instantaneous charging capacity changes with the strength of incident RF waves. Therefore, widths of the energy tunnel are different in different time periods, as depicted in Fig. 9 (c).

Given the essential differences between the energy clamp model and conventional EH model, the design of offline optimal transmission policy with varying charging capacity needs to be revisited, which is discussed in the next section.

\section{OfFline Optimal Data TRANSMission}

To maximize the system performance in terms of throughput, transmission completion time, or energy consumption, existing offline methods (e.g., water-filling based algorithms and geometrical approaches) assume that the exact energy that can be received is known prior to scheduling transmissions. However, the energy clamp model reveals that the amount of energy to be harvested by an RF-EHS is no longer predetermined but influenced by the data transmission strategy in a real time fashion. Therefore, we should jointly optimize energy utilization and energy harvest in the offline optimal data transmission scheduling.

\section{A. Properties of Offline Optimal Data Transmissions}

For an offline transmission strategy, it is assumed that an RF-EHS can accurately predict the strength of energy arriving in the future and the variation of channel quality. It is further assumed that lengths of each data transmission cycle and energy harvesting cycle are known. In other words, $E_{\max }^{i}$, $h_{i}, L_{i}$ and $U_{i}$ are random variables with pre-known values ${ }^{5}$. We consider a quasi-static fading channelwith additive white Gaussian noise for the data communications. A typical powerrate function is $r(t)=B \log \left[1+\frac{|h(t)|^{2} p(t)}{B N_{0}}\right]$, where $h(t)$ is the channel gain at time $t, B$ and $N_{0}$ are the channel bandwidth and the noise power density, respectively. In Table II, we list notations and definitions of parameters used in the paper.

In the energy clamp model, it can be proved that an offline optimal data transmission strategy has the following properties to maximize the communication throughput of an RF-EHS:

Property 1. The optimal transmission rate is a constant in each data transmission cycle.

Property 2. The optimal transmission strategy never completely deplete the energy buffer until the end of the last data transmission cycle.

Property 3. Not all energy worth to be harvested, especially when the power density in an energy harvesting cycle is low, while the length of the following data transmission cycle is much longer than the previous one.

Property 1 is a common property for existing offline optimal transmission strategies, the proof of which can refer to

\footnotetext{
${ }^{5}$ Although the offline approach is not feasible in a real system, it provides a benchmark solution as well as insights for efficient online transmission scheduling, which will discussed in Section VII.
} 
Table II: Notations

\begin{tabular}{|c|l|}
\hline Symbol & \multicolumn{1}{c|}{ Definition } \\
\hline \hline$B$ & Communication bandwidth \\
\hline$C$ & Capacitance of the energy buffer (i.e., supercapacitor) \\
\hline $\mathcal{C}(\cdot)$ & Charging function of supercapacitor \\
\hline $\mathcal{D}(\cdot)$ & A function representing relationship between $E_{r}^{1}$ and $E_{r}^{N}$ \\
\hline$E_{0}$ & Initial energy on energy buffer \\
\hline$E_{t}$ & The amount of energy stored in an energy buffer at time $t$ \\
\hline$E_{r}^{i}$ & Residual energy at the end of transmission cycle $i$ \\
\hline$E_{h}^{i}$ & Energy received during energy harvesting cycle $i$ \\
\hline$E_{\text {max }}^{i}$ & Instantaneous charging capacity of battery in harvest cycle $i$ \\
\hline $\mathcal{G}(\cdot)$ & Power-rate function \\
\hline$h_{i}$ & Channel gain in transmission cycle $i$ \\
\hline $\mathcal{L}(\cdot)$ & Lagrangian function \\
\hline$N_{0}$ & Noise power density \\
\hline$N$ & Total number of transmission cycles \\
\hline$p_{i}$ & Transmission power in transmission cycle $i$ \\
\hline$p_{i}^{*}$ & Optimal transmission power in transmission cycle $i$ \\
\hline$r_{i}$ & Transmission rate in transmission cycle $i$ \\
\hline$R$ & Resistance of charging circuit \\
\hline$S$ & Coefficient of sigmoid function \\
\hline $\mathcal{S}(\cdot)$ & Sigmoid function \\
\hline$L_{i}$ & Length of transmission cycle $i$ \\
\hline$U_{i}$ & Length of energy harvesting cycle $i$ \\
\hline$V_{\max }^{i}$ & Charging voltage in energy harvesting cycle $i$ \\
\hline$V_{t}$ & Voltage of super capacitor at time $t$ \\
\hline$\nabla_{x}(\cdot)$ & Partial derivative with respect to $x$ \\
\hline$\lambda_{i}$ & KKT multipliers \\
\hline & \\
\hline
\end{tabular}

Section III. A of [6]. This property converts continuous power management into a piecewise-linear optimization problem.

The proof of Property 2 can refer to Section 5.2 of our previous work [32]. This property arises from the nonlinear charging characteristic of the energy buffer. Property 2 indicates that the optimal transmission strategy must have an important feature: $E_{r}^{i} \neq 0$ for $i=1, \ldots, N-1$ and $E_{r}^{i}=0$ for $i=N$. This feature is important to design the offline optimal transmission strategy.

Property 3 is a new property introduced by the energy clamp model. This property is validated in Section VI-A. It reveals the tradeoff between energy utilization and energy harvest efficiency. To be specific, in order to harvest the weak energy, the RF-EHS needs to increase the transmission power (i.e., consume energy faster) to avoid energy clamp. However, the high transmission power degrades the efficiency of energy utilization according to the concave power-rate function. Therefore, instead of mandatory harvesting all received energy packets in the conventional model, whether or not to harvest the received energy becomes an option and needs to be carefully decided in transmission scheduling algorithms with the energy clamp model.

Conventional strategies hold that all energy packets can be successfully received if the energy buffer is not full and thus ignores the efficiency of energy harvest. Specifically, the profile of cumulative energy consumption (i.e., $\int_{0}^{t} p(x) \mathrm{d} x$ ) will not exceed the feasible energy tunnel [4]-[6], [8], as shown in Fig. 17(a) to guarantee that all energy will be harvested. By comparing the optimal transmission strategies

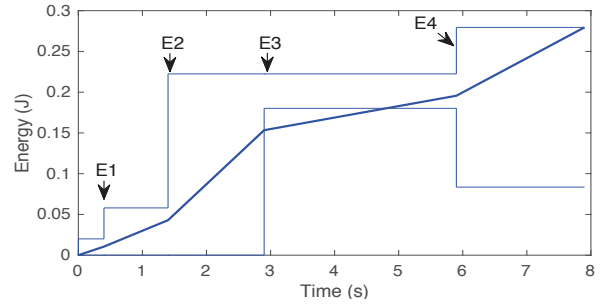

(a)

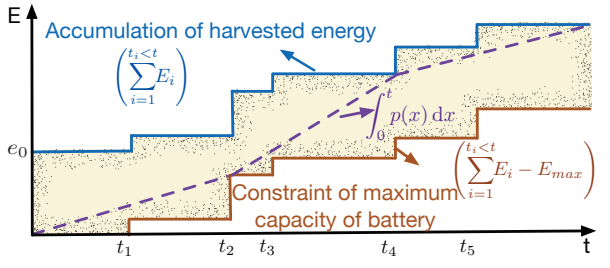

(b)

Figure 10: Optimal transmission strategy with (a) energy clamp model. (b) conventional EH model.

in Fig. 17(a) and Fig. 17(b), we can observe the impact of Property 3 on balancing the energy harvest and energy utilization efficiency with the energy clamp model.

\section{B. Offline Optimal Transmission Strategy}

The varying instantaneous charging capacity of the energy buffer due to fluctuations in RF power density in the environment makes conventional water-filling algorithms and geometrical approaches not work properly in the new energy harvesting model. To tackle this problem, we first formulate the offline data transmission as an optimization problem and then solve the problem through a recursive algorithm.

According to the piecewise linear feature of the optimal data transmission strategy introduced in Property 1, we obtain the following optimization problem with a fading channel.

$$
\begin{aligned}
& \text { P1: } \underset{p_{i}}{\arg \max } \sum_{i=1}^{N+1} \frac{L_{i} B}{2} \log _{2}\left(1+\frac{\left|h_{i}\right|^{2} p_{i}}{B N_{0}}\right), \\
& \text { S.t. } \quad i=1, \ldots, N, \\
& \text { C1: }-E_{r}^{i} \leq 0, \quad, \\
& \text { C2: } E_{r}^{i}+E_{h}^{i+1} \leq \max \left\{E_{\text {max }}^{i+1}, E_{r}^{i}\right\}, \quad i=1, \ldots, N-1,
\end{aligned}
$$

where

$$
E_{r}^{i}=E_{0}+\sum_{j=1}^{i} E_{h}^{j}-\sum_{j=1}^{i} p_{j} L_{j}, \quad i=1, \ldots, N,
$$

and the expression of $E_{h}^{i}$ can be found in (5).

The objective function in $\boldsymbol{P} \mathbf{1}$ is to find the optimal transmission power, $p_{i}$, in each data transmission cycle to maximize the communication throughput. The constraint $C l$ is an energy causality constraint, indicating that the total energy consumed by RF-EHS must not exceed the cumulative energy received in all previous harvesting cycles. $C 2$ is an energy storage constraint that specifies the instantaneous charging capacity of the energy buffer. Essentially, if $E_{r}^{i}<E_{\max }^{i+1}$, no energy clamp will occur; hence the total amount of energy that can 
be charged to the energy buffer depends on the incident power density in the current energy harvesting cycleand cannot exceed $E_{m a x}^{i+1}$. If $E_{r}^{i} \geq E_{m a x}^{i+1}$, the energy level will be forcibly clamped at $E_{r}^{i}$.

The definition of all parameters can be found in Table II. Substituting (5) into (6) to (7), we can convert the design of an efficient data transmission strategy into an optimization problem that takes into account the impact of varying power density on the energy harvesting process.

In Appendix A, we prove that constraint $C 2$ can be removed from $\boldsymbol{P 1}$ without affecting the optimal solution. Accordingly, the original optimization problem can be simplified as

$$
\begin{aligned}
& \text { P2: } \underset{p_{i}}{\arg \max } \sum_{i=1}^{N+1} \frac{L_{i} B}{2} \log _{2}\left(1+\frac{\left|h_{i}\right|^{2} p_{i}}{B N_{0}}\right), \\
& \text { s.t. }{ }_{\text {C1: }}^{i} \sum_{j=1} p_{j} L_{j}-\sum_{j=1}^{i} E_{h}^{j}-E_{0} \leq 0,
\end{aligned}
$$

where the expression of $E_{h}^{i}$ can be found in (3) and (5); the relationship between $E_{h}^{i}$ and $E_{r}^{i}$ is described in (7).

Although the optimization problem, P2, looks similar to the existing work [4], [6], [14], [17], [18], they are essentially different, as the $E_{h}^{j}$ in $\boldsymbol{P} \mathbf{2}$ is not a known value but a function of $E_{r}^{j-1}$ and $E_{\max }^{j}$. Traditional solutions that are based on conventional EH model no longer work. Therefore, we revisit the offline and online transmission policies with the energy clamp model in Section V-C and Section VII, respectively.

\section{Solutions to Optimization Problem}

Although there is only a single constraint in $\boldsymbol{P 2}$, it is not easy to solve because: (a) the step function, $E_{h}^{i}$, in (5) is not continuously differentiable, and (b) even when energy clamp does not occur, $E_{h}^{i}$ is a complex nonlinear function of $p_{i}$ according to (3) and (7), making it difficult to identify whether or not $\mathrm{Cl}$ is convex. Next, we introduce how to address those two challenges.

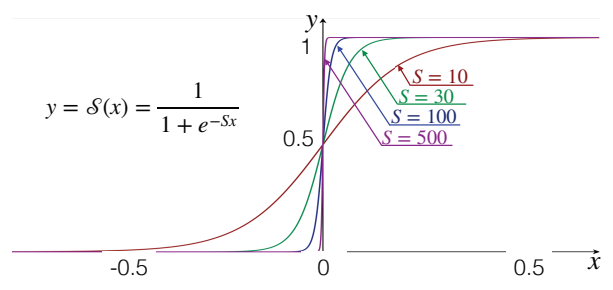

Figure 11: A set of sigmoid curves.

To find the offline optimal transmission strategy from $\boldsymbol{P 2}$, we first reform $E_{h}^{i}$ in (5) via a continuously differentiable function. This can be done by using a sigmoid function, $\mathcal{S}(x)=\frac{1}{1+e^{-S x}}$, to approximate the piecewise function. As illustrated in Fig. 11, by increasing the value of the coefficient $S, \mathcal{S}(x)$ gradually approaches Heaviside step function.
Accordingly, we can reconstruct $E_{h}^{i}$ as follows:

$$
\begin{aligned}
E_{h}^{i} & =\lim _{S \rightarrow \infty} \mathcal{C}\left(E_{r}^{i-1}, U_{i}, E_{\text {max }}^{i}\right) \cdot \mathcal{S}\left(E_{\text {max }}^{i}-E_{r}^{i}\right) \\
& =\lim _{S \rightarrow \infty} \frac{\mathcal{C}\left(E_{r}^{i-1}, U_{i}, E_{\text {max }}^{i}\right)}{1+e^{-S\left(E_{\text {max }}^{i}-E_{r}^{i}\right)}},
\end{aligned}
$$

where the charging function $\mathcal{C}\{\cdot\}$ has been introduced in (3). By using the approximate function of $E_{h}^{i}$, the constraint $C l$ in $\boldsymbol{P 2}$ becomes continuously differentiable.

From (8), we observe that $\boldsymbol{P 2}$ is a nonlinear maximization problem with inequality constraints, and the optimal solution of $\boldsymbol{P 2}$ will satisfy the Karush-Kuhn-Tucker (KKT) first-order necessary conditions. We list the stationarity, primal feasibility, and complementary slackness conditions in Appendix B.

The objective function of $\boldsymbol{P} \mathbf{2}$ is concave since its Hessian matrix is negative semidefinite for all $p_{i}$. However, it is hard to verify whether or not the left side of the constraint $\mathrm{Cl}$ is a concave function of $p_{i}$ due to the complex relationship between $p_{i}$ and $E_{h}^{i}$ described in (7), (4), and (9). Therefore, the KKT conditions are necessary but not sufficient. In this case, we may obtain multiple local optimal solutions from the KKT conditions and eliminations need to be done to find the global optimal solution.

To solve the KKT conditions, we start from the complementary slackness. According to Property 2 and (A.8), it can be obtained that for $i=1, \ldots, N-1, \sum_{j=1}^{i} p_{j}^{*} L_{j}-\sum_{j=1}^{i} E_{h}^{j}-E_{0} \neq 0$ and thus $\lambda_{i}=0$. Substituting $\lambda_{1}=\ldots=\lambda_{N-1}=0$ into (A.5), then we have that for $m=1, \ldots, N$ :

$$
\nabla_{p_{m}^{*}} \mathcal{L}=\frac{B L_{m}\left|h_{m}\right|^{2}}{2 \ln 2\left(B N_{0}+\left|h_{m}\right|^{2} p_{m}^{*}\right)}+\lambda_{N}\left(\nabla_{p_{m}^{*}} \sum_{j=1}^{N} E_{h}^{j}-L_{m}\right) .
$$

According to the relationship among harvested energy, $E_{h}^{j}$, residual energy, $E_{r}^{j}$ and transmission power, $p_{j}$ described in (7) and (9), we derive an iterative expression of $\nabla_{p_{m}^{*}} \sum_{j=1}^{m} E_{h}^{j}$ :

$$
\nabla_{p_{m}^{*}} \sum_{j=1}^{m} E_{h}^{j}=L_{m}\left[1-\prod_{j=m}^{k}\left(\mathcal{V}_{j}+1\right)\right], \quad k=m, \ldots, N-1,
$$

where $\mathcal{V}_{m} \triangleq \partial E_{h}^{m} / \partial E_{r}^{m}$. Substituting (11) into (10), it can be obtained that

$$
\nabla_{p_{m}^{*}} \mathcal{L}=L_{m}\left[\frac{B\left|h_{m}\right|^{2}}{2 \ln 2\left(B N_{0}+\left|h_{m}\right|^{2} p_{m}^{*}\right)}-\lambda_{N} \prod_{j=m}^{N-1}\left(\mathcal{V}_{j}+1\right)\right] .
$$

To satisfy the stationarity of the KKT conditions, let $\nabla_{p_{m}^{*}} \mathcal{L} \triangleq 0$ and then we have that

$$
\begin{aligned}
p_{m}^{*} & =\frac{1}{2 \lambda_{N} \ln 2 \prod_{j=m}^{N-1}\left(\mathcal{V}_{j}+1\right)}-\frac{B N_{0}}{\left|h_{m}\right|^{2}}, \\
& =\left(\frac{B N_{0}}{\left|h_{m-1}\right|^{2}}+p_{m-1}^{*}\right)\left(\mathcal{V}_{m-1}+1\right)-\frac{B N_{0}}{\left|h_{m}\right|^{2}} .
\end{aligned}
$$

In (13), we give a recursive expression of $p_{i}^{*}$, where $\mathcal{V}_{m-1}=$ 
$\partial E_{h}^{m} / \partial E_{r}^{m-1}$ can be calculated based on (3), (4), and (9). (13) is a general relationship for the optimal transmission power and can be applied to other sustainable energy resources, such as thermal, vibration, pressure energy harvesters, where the instantaneous charging voltage changes with respect to the dynamic intensity of the energy source. However, if different battery is used, the energy-voltage function (i.e., (1)) will need to be modified and (13) may not apply.

According to the relationship among $E_{h}^{i}, E_{r}^{i}$ and $p_{i}^{*}$ described in (7) and (9) and the iterative expression (13), $E_{r}^{i}$ can be represented by a nonlinear function of $p_{1}^{*}$. Utilizing Property 2 that all harvested energy will be exhausted at the end of data transmission, i.e., $E_{r}^{N}=0$, a high-order recursive equation $E_{r}^{N}=\mathcal{D}\left(p_{1}^{*}\right)=0$ is available, which can be solved via a numerical approach like Newton's method and Steffensen's method. The computational complexity solving the high-order recursive equation mainly depends on the required precision. For instance, the time complexity of the Newton's method to solve $\mathcal{D}\left(p_{1}^{*}\right)=0$ with $n$-digit precision is $\mathcal{O}(\log n) \mathcal{F}(n)$, where $\mathcal{F}(n)$ is the cost of calculating $\mathcal{D}\left(p_{1}^{*}\right) / \mathcal{D}^{\prime}\left(p_{1}^{*}\right)$.

\section{Numerical Evaluation}

In this section, we evaluate the performance of the offline optimal transmission strategy with the energy clamp phenomenon. To be specific, we compare the transmission scheduling policies with and without considering the energy clamp phenomenon.

Both transmission policies use the same objective function as illustrated in $\boldsymbol{P 1}$. The differences lie in the ways how the instantaneous charging voltage and charging capacity are treated. In the energy clamp model, dynamic charging voltage and charging capacity (i.e., $V_{\max }^{i}$ and $E_{\max }^{i}$ ) are calculated based on the strength of incident energy. By contrast, in the benchmark policy that does not consider the impact of varying RF power density on the charging voltage, constant charging voltage and charging capacity, which are denoted by $\overline{V_{\max }}$ and $\overline{E_{\max }}$ respectively, are assumed. We first analyze the differences between the behaviors of two transmission strategies in an 8-tunnel example. Afterward, the performance enhancements with the energy clamp model is presented and then some practical issues are discussed.

\section{A. Behaviors of Optimal Transmission Strategy with the en- ergy clamp model and the conventional model}

In Fig. 12, we illustrate the energy tunnel and the transmission consumption curves for the offline optimal transmission policies with the energy clamp model and the benchmark policy with the conventional model that ignores the dynamic charging voltage. The red staircase curves and the blue dash ones forms energy tunnels for the energy clamp model and the benchmark policy, respectively. The top staircase curves are the accumulative energy harvested with two strategies. The vertical distance (i.e., width of energy tunnel) between a pair of staircase curves is the dynamic charging capacity of battery.

As demonstrated in Fig. 12, after considering the varying strength of RF waves, the tunnel width in the energy clamp model changes significantly over time. The conventional model, by contrast, has a fixed tunnel width. Moreover, the end of the red step curve is higher than the end of blue one, which indicates that more energy can be harvested with the energy clamp model than the conventional one. This is because as revealed in (3) and (4), the amount of energy that can be harvested from the environment (i.e., $E_{h}^{i}$ ) depends on the instantaneous charging capacity (i.e., $E_{\max }^{i}$ ); the conventional model, however, overlooks the dynamics in $E_{\max }^{i}$ and thus has an inaccurate estimation on $E_{h}^{i}$, which in turns results in less harvested energy.

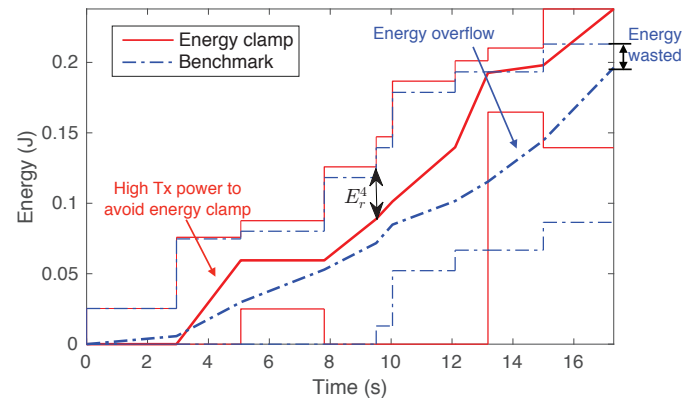

Figure 12: Transmission strategy with the energy clamp model and benchmark method.

The red and the blue piecewise linear curves in Fig. 12 indicate the accumulative energy consumption of the two transmission strategies. By comparing two transmission strategies, we observe that the accumulative energy consumption with the energy clamp model has more variations in transmission power than that with the benchmark strategy. To be specific, the energy clamp model may sharply increase the transmission power to avoid energy clampping (e.g., in $[3 \mathrm{~s} \rightarrow 5 \mathrm{~s}]$ and $[12 \mathrm{~s} \rightarrow 13 \mathrm{~s}]$ ) when the width of the incoming tunnel decreases (e.g., at $5 \mathrm{~s}$ and $13 \mathrm{~s}$ ), while reducing the transmission power (e.g., in $[5 \mathrm{~s} \rightarrow 7.9 \mathrm{~s}]$ ) when the next energy packet has high intensity (e.g., at $7.9 \mathrm{~s}$ ).

The logic behind it is that the ratio of the buffered energy, $E_{r}^{i-1}$, to the instantaneous charging capacity, $E_{m a x}^{i}$, affects the energy harvest efficiency ${ }^{6}$. As discussed in [33], according to the nonlinear charging feature of the energy buffer, the harvester achieves the highest harvest efficiency around $E_{r}^{i-1} / E_{\max }^{i}=0.25$. However, it is worth noting that more harvested energy does not guarantee a higher throughput, as $E_{r}^{i-1}$ also influences the efficiency of energy utilization ${ }^{7}$ as shown in (7). Therefore, the optimal transmission strategy seeks a tradeoff between the energy harvest efficiency and energy utilization efficiency.

By contrast, the transmission power shows less changes in the benchmark strategy. By assuming constant charging voltage, the variation of tunnel width is overlooked. The energy tunnel bounded by the blue dash staircase curves actually does

\footnotetext{
${ }^{6}$ The energy harvest efficiency characterizes the ability of the EH system to actually capture the energy at a given intensity of arrival energy.

${ }^{7}$ The energy utilization efficiency as the ratio of transmitted data to the amount of harvested energy, $\sum_{i} E_{h}^{i}$ characterizes how efficient the EH system utilize the harvested energy.
} 
not exist in the real world. Therefore, the capacity constraint of energy buffer in the benchmark transmission scheduling cannot fully avoid the energy overflow. Especially when the instantaneous charging capacity reduces in the environment (e.g., at $13.1 \mathrm{~s}$ in Fig. 12), energy overflows are highly likely to incur and cause low energy harvest efficiency. In addition to possible energy overflow, the conventional strategy also has lower utilization efficiency than the energy clamp model. The transmission policies are supposed to utilize all the cumulative energy at the end of the experiment to avoid energy waste. However, owing to the unrealistic assumption on the instantaneous charging capacity, the benchmark policy won't be able to accurately estimate the amount of harvested energy, $E_{h}^{i}$. As shown in Fig. 12, in the 8-tunnel example, there is energy left over when the test ends at $17 \mathrm{~s}$. The wasted energy further reduces the performance of the benchmark strategy.

\section{B. Performance Comparison}

In the following evaluations, we will verify that the transmission strategy with the energy clamp model has superior performance in terms of harvested energy and throughput compared to that with the conventional model.
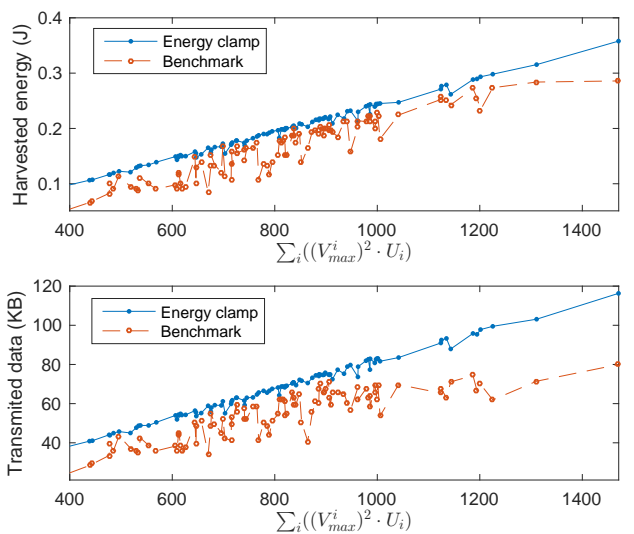

Figure 13: Harvested energy and throughput with respect to accumulative energy arrivals.

Fig. 13 illustrates the amount of harvested energy and the amount of transmitted data of two strategies in 100 independent tests. The corresponding charging voltage, $V_{\text {max }}^{i}$, for each energy packet has a uniform distribution with $2.25 \mathrm{~V}$ mean and $20 \%$ relative standard deviation ${ }^{8}$. The length of energy packet in seconds also has a uniform distribution, $U_{i} \sim U(5,30)$.

We define the energy harvest efficiency as the ratio of the amount of harvested energy, $\sum_{i} E_{h}^{i}$, to the efficient energy that reaches battery. It characterizes the ability of the EH system to actually capture the energy and store the energy in battery. From the $V_{\max }^{i}$ and $P_{e}^{i}$ relationship demonstrated in Fig. 3, we observe that when $P_{e}^{i}$ is between $0.05 \mathrm{~mW}$ and $0.5 \mathrm{~mW}$ (i.e., $-14.5 \mathrm{dBm}$ and $-1 \mathrm{dBm}),\left(V_{\max }^{i}\right)^{2}$ is nearly proportional to $P_{e}^{i}$. Therefore, we use $\sum_{i} U_{i}\left(V_{\max }^{i}\right)^{2}$, as an

\footnotetext{
${ }^{8}$ Relative standard deviation, also called coefficient of variation, is defined as the ratio of standard deviation $\sigma$ to the mean $\mu$.
}

indicator to represent the efficient energy reached the energy buffer. The indicator of energy harvest efficiency, (i.e., yaxis of Fig. 14) is thus calculated as the ratio of $\sum_{i} E_{h}^{i}$ to $\sum_{i} U_{i}\left(V_{\max }^{i}\right)^{2}$. Due to the randomness of $V_{\max }^{i}$ and $U_{i}$, $\sum_{i} U_{i}\left(V_{\max }^{i}\right)^{2}$ has a vast variation, ranging from 400 to 1, 500, among different tests. Therefore, the $\mathrm{x}$-axis of Fig. 13 and Fig. 14 also indicates the variation of energy intensity: the further $\sum_{i} U_{i}\left(V_{\max }^{i}\right)^{2}$ deviates from the average (i.e., around 900), the larger variation $V_{\max }^{i}$ has. In some tests when $\sum_{i} U_{i}\left(V_{\max }^{i}\right)^{2}$ is between 700 and 1,000 , the variation of $V_{\text {max }}^{i}$ is moderate. When $\sum_{i} U_{i}\left(V_{\text {max }}^{i}\right)^{2}$ is below 600 or above 1,200, $V_{\max }^{i}$ has significant variations; this is also the scenario when the energy clamp model and the conventional model have large performance gap.
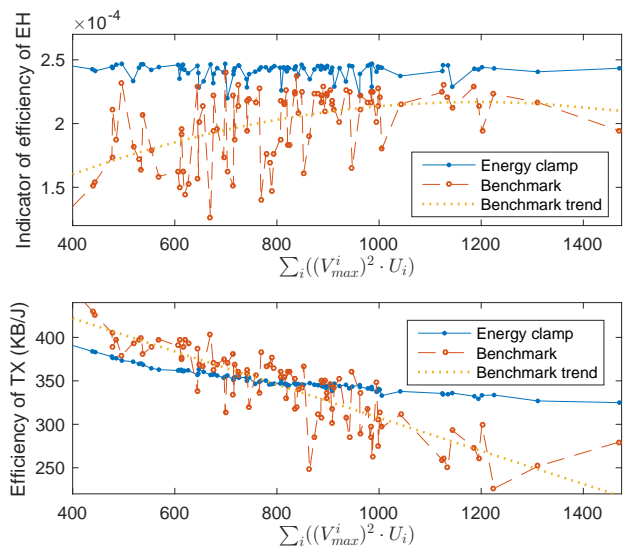

Figure 14: Efficiency of energy harvest and energy utilization with accumulative energy arrivals.

The transmission strategy with the energy clamp model is able to adapt to the dynamics of incident RF power and achieve linear performance growth when more energy is available in the air, which indicates a stable efficiency of energy harvest as shown in the top figure of Fig. 14. By contrast, the benchmark strategy with conventional model assumes constant $V_{\max }$ and has inaccurate estimation on the amount of energy that can be truly harvested. It, therefore, achieves much lower energy harvest efficiency than the energy clamp model as shown in the top figure of Fig. 14.

We define the energy utilization efficiency as the ratio of transmitted data to the amount of harvested energy, $\sum_{i} E_{h}^{i}$, in the offline optimal transmission policy. It characterizes how efficient the EH system utilize the harvested energy. According to Property 1 and its proof in [6], a good energy utilization efficiency is generally achieved when the transmission power is low and has least variation. As demonstrated in Fig. 12 of the manuscript, in order to adapt to the dynamics of incident RF power, the transmission power needs to adjust accordingly to avoid energy clamp phenomenon. Therefore, the transmission power in energy clamp model has more variations than that in the conventional EH model as shown in the bottom figure of Fig. 14. As a result, the energy utilization efficiency of conventional policies is higher when $\sum_{i} U_{i}\left(V_{\text {max }}^{i}\right)^{2}$ is less 
than 900 (i.e., with small $V_{\max }^{i}$ the energy waste less occurs).

However, higher energy utilization efficiency cannot guarantee higher throughput since the lower energy harvest efficiency may compensate for the benefit of higher utilization efficiency. One example is the transmission policy in conventional $\mathrm{EH}$ model. Although when $\sum_{i} U_{i}\left(V_{\text {max }}^{i}\right)^{2}$ is less than 900 , it achieves higher utilization efficiency than the energy clamp model, its energy harvest efficiency is much lower due to its inaccurate estimation on $E_{h}^{i}$ under the impact of varying $V_{\text {max }}^{i}$. When the $E_{h}^{i}$ is underestimated (i.e., $\sum_{i} U_{i}\left(V_{\max }^{i}\right)^{2}$ is larger than 900), the energy waste occurs in an often manner and the offline optimal transmission policy in the energy clamp model outperforms that in conventional EH model in terms of both energy harvest efficiency and energy utilization efficiency.

Note that the performance gap between the energy clamp model and the conventional model increases when the $V_{\max }$ has a high variation (i.e., low or high $\sum_{i} U_{i}\left(V_{\text {max }}^{i}\right)^{2}$ in Fig. 13). The reason is obvious: the larger the deviation of $V_{\max }^{i}$ from the mean value, the worse the traditional model's estimate of the harvested energy. It, in turn, leads to worse throughput. The same phenomenon is also revealed in Fig. 15. In the test, we change the deviation and mean value of $V_{\max }^{i}$ to evaluate their impact on the performance enhancement with the energy clamp model.

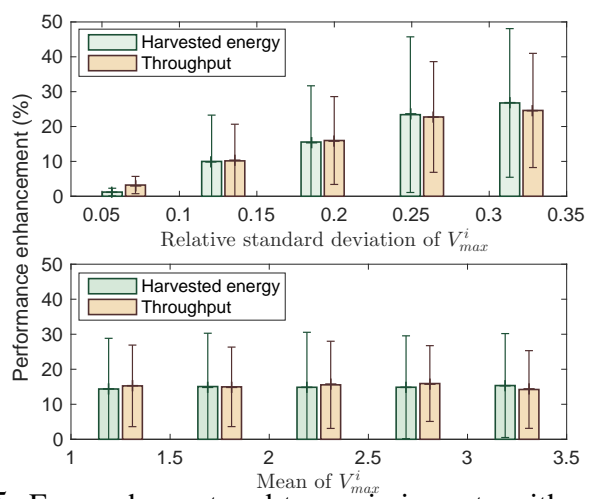

Figure 15: Energy harvest and transmission rate with respect to the standard deviation of incident RF energy.

In the top figure of Fig. 15, the mean of $V_{\max }^{i}$ is $2.25 \mathrm{~V}$ and the relative standard deviation varies from $6 \%$ to $32 \%$. Each errorbar plot comes from 100 independent tests. As shown in the figure, regardless the significance of deviation, the transmission strategy with the energy clamp model always have positive performance improvement in terms of harvested energy and throughput than the benchmark strategy with the conventional model. The performance enhancement increases with the growth of $V_{\max }^{i}$ 's deviation. As revealed in Fig. 7, the relative standard deviation of charging voltage tested in real experiment is $17.9 \%$ with $1 \mathrm{dBi}$, which could lead to about $16 \%$ performance enhancement with the energy clamp model.

We also varied the mean value of the $V_{\max }^{i}$ and demonstrate the performance enhancement with the energy clamp model in the bottom figure. In these tests, the relative standard deviation was fixed as $20 \%$. As shown in Fig. 7, the varying average strength of RF waves has no obvious impact on the performance difference.

From the above analysis, we can conclude that 1) conventional models fail to accurately estimate the amount of harvested energy, which in turns degrades the throughput performance of existing transmission strategies; 2) the transmission strategy with the energy clamp model is able to adapt to highly dynamic RF strength and achieves linear performance growth there is more energy in the air; 3) the transmission strategy with the energy clamp model always achieves higher throughput than that with the conventional model; and 4) the significance of performance enhancement grows with the growing fluctuations of the signal strength.

\section{ONLINE TRANSMisSion SCHEDULING}

Although the offline optimal policy can achieve the highest throughput under the limited energy constraints, it is not feasible in practice due to two essential limitations. First, it's non-causal as it assumes to know all the future energy arrivals. Second, as the length of feasible energy tunnel grows, the computation complexity of the nonlinear optimization problem in Section V-C increases significantly. Following these concerns, we investigate the possibility of dividing long tunnels into shorter ones and using prediction to assist in the online transmission scheduling.

In the simulation evaluation, we assume that the original length of the energy tunnel, $N$, is 64 . Instead of scheduling the transmissions in the 64-tunnel at one time, we break the 64-tunnel into shorter ones and seek for individual optimal transmission strategy in each sub-tunnel. A suboptimal solution will be obtained by combining the transmission strategies in all sub-tunnels. In this transition, we expect a performance degradation compared to the original optimal solution. Considering the ultra-low energy harvest efficiency when the energy buffer is completely drained, we arrange an amount of energy left over at the end of each sub-tunnel. The residual energy, $E_{r}$, in the previous period will be the initial energy, $\hat{E}_{0}$, of energy buffer in the following sub-tunnel.

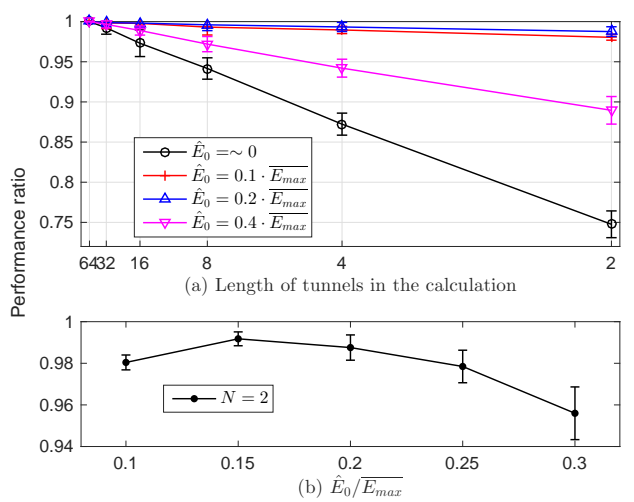

Figure 16: Performance degradation when using shorter tunnel.

Fig. 16 (a) shows the performance degradations when we divide the long 64-tunnel into shorter ones (i.e., $N=32,16,8,4$ and 2). Take $N=2$ on the blue curve as an example. Since we 
set a constant $\hat{E}_{0}=0.2 \overline{E_{\max }}$, the residual energy at the end of each sub-tunnel (i.e., $E_{r}^{2 \cdot i}, i=1, \ldots, N / 2$ ) will be fixed to $0.2 \overline{E_{\max }}$. However, by observing Fig. 12 , we can see that the residual energy, $E_{r}^{k}$, varies in the offline optimal transmission strategy. The diverge of $\hat{E}_{0}$ from $E_{r}^{k}$ leads to the performance degradation. Therefore, the value of $\hat{E}_{0}$ has significant influence on the performance of the suboptimal solution. As shown in Fig. 16(b), for sub-tunnels with $N=2$, the performance degradation is less than $1 \%$ when $\hat{E}_{0}$ is around $0.15 \overline{E_{\max }}$. This result confirms the possibility of dividing long tunnels into shorter ones without much performance degradation in the offline optimal transmission scheduling.

By reviewing the behaviors of offline optimal transmission policy in the energy clamp model illustrated in Fig. 12, we observe that the optimal transmission power, $p_{i}^{*}$, depends more on the charging voltage of the next harvesting cycle, $V_{\max }^{i+1}$, than on the current charging voltage. The system needs to increase the energy consumption rate (i.e., higher transmission power) to avoid energy clamp if the next charging voltage will reduce, while reducing the transmission power for better energy harvest and utilization efficiency when the next charging voltage will increase.

The feasibility of dividing long tunnels into shorter ones (i.e., $N=2$ ) and the dependences of the current transmission power on the incoming charging voltage advocate the prediction based online transmission strategy. The prediction based online policy makes the online transmission scheduling every 2 tunnels. It calculates the transmit power (i.e., $p_{1}^{*}$ and $p_{2}^{*}$ ) according to the observed battery energy (i.e., $E_{r}^{1}$ ), the current energy arrival (i.e., $E_{h}^{1}$ ) and the estimated incoming charging voltage (i.e., $V_{\max }^{2}$ ). According to (13) and (5), $p_{2}^{*}$ and the next energy arrival (i.e., $E_{h}^{2}$ ) can be represented by $p_{1}^{*}$. Instead of drain the battery at the end of $N^{t h}$ tunnel in the offline policy, we left $0.15 \overline{E_{\max }}$ amount of energy in the battery at the end of the $2^{\text {nd }}$ tunnel. This online transmission power decision is thus converted to a Quadratic equation of $p_{1}^{*}$ that can be easily solved using numerical methods.

By leveraging the weak correlation among energy packets [34], it is feasible to predict the strength of RF waves in the near future. Next, we use a simple autoregressive (AR) [35] model of order 4 as the one-step-ahead predictor. In the simulation, we use the experimental data demonstrated in Fig. 7 to generate a series of practical dynamic charging voltage for Powercast energy harvester. Without loss of generality, we assume that the charging voltage does not change within one harvest cycle but varies among different cycles as shown in Fig. 17 (a). The relative standard deviation of $V_{\max }$ is $16.4 \%$, slightly lower than the original measured charging voltage. The 4-order AR (i.e., AR-4) model shown in (14) is applied to predict the incoming charging voltage, $V_{\max }^{2}$. Next, we conduct numerical evaluations to verify the feasibility of prediction based online transmission strategy when energy clamp phenomenon is considered.

$$
V_{\text {max }}^{i}=-\sum_{j=1}^{p} a_{j} V_{\max }^{i-j}+w[i] .
$$

The AR-4 model based one-step-ahead predictor achieves $7.6 \%$ normalized root mean-square error (RMSE). The corresponding performance gap compared with the offline optimal approach is about $0.5 \%$. With more advanced prediction methods such as In order to evaluate the performance degradation in terms of throughput and harvested energy resulted from the errors in the prediction, we manually added prediction errors ranging from $1 \%$ to $14 \%$ and showed the results in Fig. 17 (b). As displayed in the figure, compared to the offline optimal solution, the performance degrades by $0.1 \%$ to $3 \%$ when prediction error is changed from $1 \%$ to $14 \%$. The low performance degradation of AR-4 based online policy verifies the feasibility of prediction based online suboptimal transmission strategy in reality.

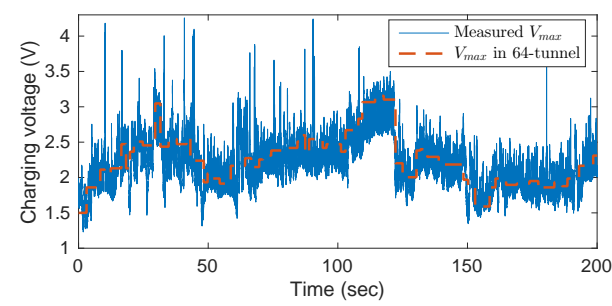

(a) Example of charging voltage in evaluation

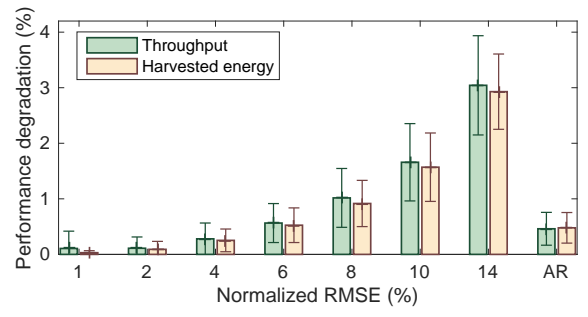

(b) Performance degradation with prediction error

Figure 17: Performance evaluation of online transmission strategy.

\section{CONCLUSION}

In this work, we revealed the phenomenon of varying charging voltage in the RF energy harvesting system that is caused by the dynamic RF strength in the environment. We proposed an energy clamp model to accurately describe the variations in the charging voltage and instantaneous charging capacity. Based on the new energy clamp model, the offline optimal transmission scheduling is proposed. Due to the nonlinear charging feature of energy buffer and the dynamic charging voltage, the amount of energy to be harvested is not predetermined but varies with the data transmission strategy. Therefore, we proposed a new offline optimal transmission scheduling strategy that jointly optimize energy harvest and energy utilization. Simulation results have demonstrated that proposed offline optimal transmission scheduling with the energy clamp model well adapts to the dynamic RF environment and achieves higher performance than the conventional strategies. The research of this paper is expected to shed light on the future research of practical energy harvesting powered wireless communications. 


\section{AcKnOWlegement}

This work is supported in part by the US National Science Foundation under Grant No. 2051356, European Research Council (ERC) project AGNOSTIC under grant 742648 and the FNR CORE project ROSETTA under grant 11632107.

\section{APPENDIX}

\section{A. Simplifying Optimization Problem P1}

Here, we prove that the constraint $C 2$ in the optimization problem $\boldsymbol{P 1}$ can be removed if an RF-EHS selects a supercapacitor as its energy buffer. This can be achieved by proving that any transmission strategy can naturally meet $C 2$ if the charging function is (3).

Proof. We first rewrite $C 2$ in (6) as follows:

$$
E_{h}^{i+1}+E_{r}^{i} \leq \max \left\{E_{\text {max }}^{i+1}, E_{r}^{i}\right\}, i=1, \ldots, N-1 .
$$

Next, we investigate two cases separately: (a) $E_{r}^{i} \geq E_{\max }^{i+1}$ and (b) $E_{r}^{i}<E_{\max }^{i+1}$.

Case 1: If $E_{r}^{i} \geq E_{\text {max }}^{i+1}, \max \left\{E_{\text {max }}^{i+1}, E_{r}^{i}\right\}=E_{r}^{i}$ and $E_{h}^{i+1}=0$ according to (5). In this case, (A.1) becomes $E_{r}^{i} \leq E_{r}^{i}$. Apparently, any data transmission strategy satisfies that condition.

Case 2: If $E_{r}^{i}<E_{\text {max }}^{i+1}, \max \left\{E_{\text {max }}^{i+1}, E_{r}^{i}\right\}=E_{\text {max }}^{i+1}$ and $E_{h}^{i+1}=\mathcal{C}\left(E_{r}^{i}, U_{i+1}, E_{\text {max }}^{i+1}\right)$. (A.1) becomes

$$
\mathcal{C}\left(E_{r}^{i}, U_{i+1}, E_{\text {max }}^{i+1}\right)+E_{r}^{i} \leq E_{\text {max }}^{i+1}
$$

Let $\frac{U_{i+1}}{R C} \triangleq \beta$, then substituting (3) and (4) into (A.2), the left side of (A.2) is

$$
\begin{aligned}
& \mathcal{C}\left(E_{r}^{i} U_{i+1}, E_{\text {max }}^{i+1}\right)+E_{r}^{i} \\
= & A_{i+1}^{1}\left(A_{i+1}^{2}\right)^{2}+A_{i+1}^{1} A_{i+1}^{3}\left(E_{r}^{i}\right)^{\frac{1}{2}}+A_{i+1}^{1} A_{i+1}^{4}\left(E_{r}^{i}+1\right) \\
= & \left(1-e^{-\beta}\right) E_{\text {max }}^{i+1}+2\left(e^{-\beta}-e^{-2 \beta}\right)\left(E_{\max }^{i+1} E_{r}^{i}\right)^{\frac{1}{2}}+e^{-2 \beta} E_{r}^{i} \\
= & {\left[e^{-\beta}\left(E_{r}^{i}\right)^{\frac{1}{2}}+\left(1-e^{-\beta}\right)\left(E_{\max }^{i+1}\right)^{\frac{1}{2}}\right]^{2} . }
\end{aligned}
$$

Substituting (A.3) into (A.2) and performing square root operations on both sides of the inequality, we get

$$
\begin{aligned}
& {\left[e^{-\beta}\left(E_{r}^{i}\right)^{\frac{1}{2}}+\left(1-e^{-\beta}\right)\left(E_{\max }^{i+1}\right)^{\frac{1}{2}}\right] \leq\left(E_{\max }^{i+1}\right)^{\frac{1}{2}} } \\
\Rightarrow & e^{-\beta}\left(E_{r}^{i}\right)^{\frac{1}{2}} \leq e^{-\beta}\left(E_{\max }^{i+1}\right)^{\frac{1}{2}} \\
\Rightarrow & \left(E_{r}^{i}\right)^{\frac{1}{2}} \leq\left(E_{\max }^{i+1}\right)^{\frac{1}{2}} .
\end{aligned}
$$

For any transmission strategy, the inequality in (A.4) is always true since $E_{r}^{i}<E_{\max }^{i+1}$.

According to the above proof, it can be realized that constraint $C 2$ in $\boldsymbol{P 1}$ is always satisfied if the charging function of an energy buffer is (3). Therefore, $C 2$ is redundant and we can remove it from the optimization problem without affecting the optimal solution.

\section{B. KKT conditions of Problem P2}

\section{Stationarity:}

$$
\begin{aligned}
\nabla_{p_{m}^{*}} \mathcal{L} & =\nabla_{p_{m}^{*}}\left(\sum_{i=1}^{N+1} \frac{L_{i} B}{2} \log _{2}\left(1+\frac{\left|h_{i}\right|^{2} p_{i}^{*}}{B N_{0}}\right)\right) \\
& -\sum_{i=1}^{N} \lambda_{i} \nabla_{p_{m}^{*}}\left(\sum_{j=1}^{i} p_{j}^{*} L_{j}-\sum_{j=1}^{i} E_{h}^{j}-E_{0}\right), m=1, \ldots, N
\end{aligned}
$$

where $\mathcal{L}$ is the Lagrangian depending on $p_{i}, \lambda_{i}$ and $\mu_{i} ; \nabla_{x}(\cdot)$ represents the partial derivative with respect to $x$, and $m=$ $1, \ldots, N+1$.

\section{Primal feasibility:}

$$
\sum_{j=1}^{i} p_{j}^{*} L_{j}-\sum_{j=1}^{i-1} E_{h}^{j}-E_{0} \leq 0, \quad i=1, \ldots, N .
$$

Dual feasibility: $\quad \lambda_{i} \geq 0, \quad i=1, \ldots, N$.

Complementary slackness:

$$
\lambda_{i}\left(\sum_{j=1}^{i} p_{j}^{*} L_{j}-\sum_{j=1}^{i} E_{h}^{j}-E_{0}\right)=0, \quad i=1, \ldots, N .
$$

\section{REFERENCES}

[1] S. Bi, Y. Zeng, and R. Zhang, "Wireless powered communication networks: an overview," IEEE Wireless Communications, vol. 23, no. 2, pp. 10-18, 2016.

[2] M. Piñuela, P. D. Mitcheson, and S. Lucyszyn, "Ambient RF energy harvesting in urban and semi-urban environments," IEEE Transactions on Microwave Theory and Techniques, vol. 61, pp. 2715-2726, 2013.

[3] Y. Luo, L. Pu, G. Wang, and Y. Zhao, "RF Energy Harvesting Wireless Communications: RF Environment, Device Hardware and Practical Issues," Sensors, vol. 19, no. 13, p. 3010, 2019.

[4] J. Yang and S. Ulukus, "Optimal packet scheduling in an energy harvesting communication system," IEEE Transactions on Communications, vol. 60, no. 1, pp. 220-230, 2012.

[5] Y. Luo, L. Pu, Y. Zhao, G. Wang, and M. Song, "DTER: optimal two-step dual tunnel energy requesting for RF-based energy harvesting system," IEEE Internet of Things Journal, pp. 1-11, 2018.

[6] K. Tutuncuoglu and A. Yener, "Optimum transmission policies for battery limited energy harvesting nodes," IEEE Transactions on Wireless Communications, vol. 11, no. 3, pp. 1180-1189, 2012.

[7] Y. Dong, F. Farnia, and A. Özgür, "Near optimal energy control and approximate capacity of energy harvesting communication," IEEE Journal on Selected Areas in Communications, vol. 33, no. 3, pp. 540557, 2015.

[8] A. Biason and M. Zorzi, "On the effects of battery imperfections in an energy harvesting device," in Proceedings of International Conference on Computing, Networking and Communications. IEEE, 2016, pp. 1-7.

[9] Y. Luo, L. Pu, Y. Zhao, W. Wang, Q. Yang, and Z. Peng, "Revisiting transmission scheduling in RF energy harvesting wireless communications," in Proceedings of the ACM International Symposium on Mobile Ad Hoc Networking and Computing (MobiHoc), 2018, pp. 314-315.

[10] Y. Luo and L. Pu, "Practical issues of RF energy harvest and data transmission in renewable radio energy powered IoT," IEEE Transactions on Sustainable Computing, 2020.

[11] Y. Luo, L. Pu, Y. Zhao, W. Wang, and Q. Yang, “A nonlinear recursive model based optimal transmission scheduling in RF energy harvesting wireless communications," IEEE Transactions on Wireless Communications, vol. 19, no. 5, pp. 3449-3462, 2020.

[12] S. Ulukus, A. Yener, E. Erkip, O. Simeone, M. Zorzi, P. Grover, and K. Huang, "Energy harvesting wireless communications: a review of recent advances," IEEE Journal on Selected Areas in Comm., vol. 33, no. 3, pp. 360-381, 2015. 
[13] X. Lu, P. Wang, D. Niyato, D. I. Kim, and Z. Han, "Wireless networks with rf energy harvesting: A contemporary survey," IEEE Communications Surveys \& Tutorials, vol. 17, no. 2, pp. 757-789, 2014.

[14] O. Ozel, K. Tutuncuoglu, J. Yang, S. Ulukus, and A. Yener, "Transmission with energy harvesting nodes in fading wireless channels: optimal policies," IEEE Journal on Selected Areas in Communications, vol. 29, no. 8, pp. 1732-1743, 2011.

[15] I. Chaour, A. Fakhfakh, and O. Kanoun, "Enhanced passive RF-DC converter circuit efficiency for low RF energy harvesting," Sensors, vol. 17, no. 3, p. 546, 2017.

[16] M. Gorlatova, A. Wallwater, and G. Zussman, "Networking low-power energy harvesting devices: measurements and algorithms," IEEE Transactions on Mobile Computing, vol. 12, no. 9, pp. 1853-1865, 2013.

[17] O. Orhan, D. Gunduz, and E. Erkip, "Energy harvesting broadband communication systems with processing energy cost," IEEE Transactions on Wireless Communications, vol. 13, no. 11, pp. 6095-6107, 2014.

[18] B. Devillers and D. Gündüz, "A general framework for the optimization of energy harvesting communication systems with battery imperfections," Journal of Communications and Networks, vol. 14, no. 2, pp. 130-139, 2012.

[19] Agilent Technologies, Agilent Technologies 8648A/B/C/D Signal Generator Operation and Service Guide, Agilent Technologies, Santa Rosa, CA, U.S.A., March 2001. [Online]. Available: https://www.naic.edu/ phil/hardware/synth/hp8648/ agilent8648OperationAndServiceGuide.pdf

[20] Powercast, P2110B $915 \mathrm{MHz}$ RF Powerharvester Receiver, Powercast Corporation, Pittsburgh, PA, U.S.A., July 2015.

[21] Keysight Technologies, Keysight N9340B Handheld Spectrum Analyzer User's Guide, Keysight Technologies, Santa Rosa, CA, U.S.A., December 2015. [Online]. Available: https://literature.cdn.keysight.com/ litweb/pdf/N9340-90008.pdf

[22] P. Nintanavongsa, U. Muncuk, D. Lewis, and K. Chowdhury, "Design optimization and implementation for RF energy harvesting circuits," IEEE Journal on emerging and selected topics in circuits and systems, vol. 2, no. 1, pp. 24-33, 2012.

[23] G. Papotto, F. Carrara, and G. Palmisano, "A 90-nm CMOS thresholdcompensated RF energy harvester," IEEE Journal of Solid-State Circuits, vol. 46, no. 9, pp. 1985-1997, 2011.

[24] H. Jabbar, Y. S. Song, and T. T. Jeong, "RF energy harvesting system and circuits for charging of mobile devices," IEEE Transactions on Consumer Electronics, vol. 56, no. 1, 2010.

[25] Keysight Technologies, Keysight N6850A Broadband Omnidirectional Antenna, Keysight Technologies, Inc., Santa Rosa, CA, December 2015.

[26] Cellmapper, "Cellular Tower and Signal Map," cellmapper.net, [Accessed: Nov., 2019]. [Online]. Available: https://www.cellmapper.net/map?MCC $=310 \& M N C=410 \&$ type $=$ LTE\&latitude $=33.45259377630629 \&$ longitude $=-88.7879499000566$

[27] K. Kaemarungsi and P. Krishnamurthy, "Analysis of WLAN's received signal strength indication for indoor location fingerprinting," Pervasive and mobile computing, vol. 8, no. 2, pp. 292-316, 2012.

[28] Panasonic Solar Amorton Co., Ltd., "Panasonic Amorphous Silicon Solar Cells Catalog," https://panasonic.co.jp, 2019, [Accessed: Dec., 2019]. [Online]. Available: https://panasonic.co.jp/ls/psam/en/products/ pdf/Catalog_Amorton_ENG.pdf

[29] S. Niu, X. Wang, F. Yi, Y. S. Zhou, and Z. L. Wang, "A universal selfcharging system driven by random biomechanical energy for sustainable operation of mobile electronics," Nature communications, vol. 6, 2015

[30] Z. Lu, H. Zhang, C. Mao, and C. M. Li, "Silk fabric-based wearable thermoelectric generator for energy harvesting from the human body," Applied Energy, vol. 164, pp. 57-63, 2016.

[31] J. W. Nilsson and S. Riedel, Electric Circuits, 2015.

[32] Y. Luo, L. Pu, Y. Zhao, W. Wang, and Y. Qing, "Revisiting transmission scheduling in rf energy harvesting wireless communications," arXiv preprint arXiv:1802.09328v1, 2018.

[33] Y. Luo, L. Pu, Y. Zhao, G. Wang, and M. Song, "Optimal energy requesting strategy for RF-based energy harvesting wireless communications," in Proceedings of the IEEE Conference on Computer Communications (INFOCOM), 2017, pp. 1-9.

[34] P. Blasco, D. Gunduz, and M. Dohler, "A learning theoretic approach to energy harvesting communication system optimization," IEEE Trans. on Wireless Communications, vol. 12, no. 4, pp. 1872-1882, 2013.

[35] K. E. Baddour and N. C. Beaulieu, "Autoregressive modeling for fading channel simulation," IEEE Transactions on Wireless Communications, vol. 4, no. 4, pp. 1650-1662, 2005.

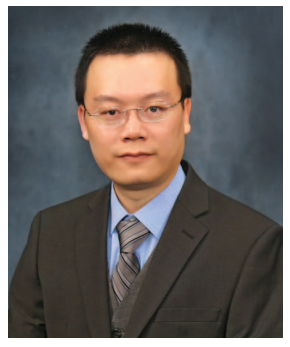

Dr. Yu Luo received the B.S. degree and the M.S. degree in electrical engineering from the Northwestern Polytechnical University, China, in 2009 and 2012, respectively. In 2015, he received the $\mathrm{Ph} . \mathrm{D}$. degree in computer science and engineering from University of Connecticut, Storrs. Dr. Luo is currently an Assistant Professor at Mississippi State University. His major research focus on the sustainable wireless networks for emerging IoT, RF energy harvesting hardware, security in RF energy harvesting wireless networks, and underwater wireless networks. $\mathrm{He}$ is a Co-recipient of the Best Paper Award in IFIP Networking 2013 and Chinacom 2016.

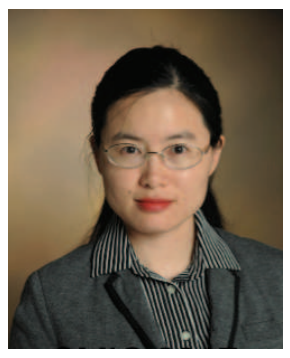

Dr. Lina Pu received the B.S. degree in electrical engineering from the Northwestern Polytechnical University, Xi'an, China in 2009 and the Ph.D. degree in Computer Science and Engineering from University of Connecticut, Storrs. Dr. Pu is currently an Assistant Professor at University of Southern Mississippi. Her research interests lie in the area of RF energy harvesting wireless networks, security in the sustainable IoT, and underwater acoustic/VL networks. She owned IFIP Networking 2013 best paper award.

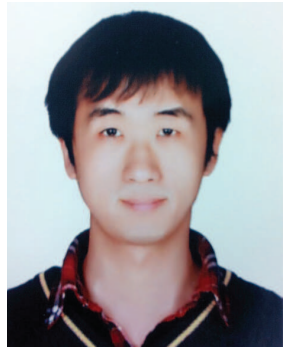

Dr. Lei Lei (S'12-M'17) is currently a Research Scientist at the Interdisciplinary Centre for Security, Reliability and Trust (SnT), University of Luxembourg. He received the B.Eng. and M.Eng. degrees from Northwestern Polytechnic University, Xi'an, China, in 2008 and 2011, respectively. He obtained his Ph.D. degree in 2016 at the Department of Science and Technology, Linköping University, Sweden. He joined SnT as a research associate in Nov. 2016. He was a research assistant at Institute for Infocomm Research (I2R), A*STAR, Singapore, from June 2013 to December 2013. His current research interests include resource allocation and optimization in 5G-satellite networks, energy-efficient communications, and deep learning in wireless communications. He received the IEEE Sweden Vehicular Technology-Communications-Information Theory joint chapter best student journal paper award in 2014. He was a co-recipient of the IEEE SigTelCom'19 Best Paper Award. 